\title{
Recent progress in higher order computations for the physics of the Higgs boson
}

H. van Deurzen, N. Greiner, G. Luisoni, E. Mirabella, T. Peraro, J.F. von

Soden-Fraunhofen, J. Winter, V. Yundin

Max Planck Institute for Physics, Föhringer Ring 6, 80805 Munich, Germany

E-mail: \{hdeurzen, greiner, luisonig, mirabell, peraro, jfsoden, jwinter, yundin\} ampp.mpg.de

\section{J. Huston}

Michigan State University, East Lansing, MI USA 48824

E-mail: huston@msu.edu

\section{P. Mastrolia}

Max Planck Institute for Physics, Föhringer Ring 6, 80805 Munich, Germany;

Dipartimento di Fisica e Astronomia, Università di Padova, and INFN Sezione di Padova, via Marzolo 8, 35131 Padova, Italy

E-mail: ppaolo@mpp.mpg.de

\section{G. Ossola}

Physics Department, New York City College of Technology, The City University of New York, 300 Jay Street Brooklyn, NY 11201, USA;

The Graduate School and University Center, The City University of New York, 365 Fifth Avenue, New York, NY 10016, USA

E-mail: gossola@citytech. cuny .edu

\section{F. Tramontano*}

Dipartimento di Scienze Fisiche, Università degli studi di Napoli and INFN, Sezione di Napoli, 80126 Napoli, Italy

E-mail: francesco.tramontanodcern.ch

We have computed the next to leading order QCD correction to the production of an Higgs boson in association with up to three jets in gluon fusion. We have worked in the heavy top mass limit. Preliminary results for event rates and distributions at the LHC energies are shown.

Loops and Legs in Quantum Field Theory - LL 2014,

27 April - 2 May 2014

Weimar, Germany

\footnotetext{
* Speaker.
} 


\section{Introduction}

In the Standard Model of the elementary particles, the gluon fusion mechanism (GGF) gives the largest total cross section for Higgs boson $(H)$ production in hadron-hadron collisions. This production channel gives an indirect measurement of the strength of the interaction among the Higgs boson and the fermions. Further, the precise knowledge of Higgs boson production rates in association with jets via gluon fusion allows to disentangle this channel from vector boson fusion production, where the Higgs boson is radiated off electroweak gauge bosons. On the other hand, more exclusive search strategies based on the use of a jet veto require the detailed knowledge of differential rates for the production of Higgs plus jets.

Rates for Higgs boson production in association with two and three jets, retaining the full top-mass $\left(m_{t}\right)$ dependence, have been computed respectively in Refs. [1,2], and Ref. [3]. These calculations showed that the large top-mass approximation $\left(m_{t} \rightarrow \infty\right)$ is valid whenever the mass of the Higgs particle and the $p_{T}$ of the jets are not much larger than the mass of the top quark. The next-to-leading order (NLO) corrections for $H+2$-jets in GGF were first computed in Ref. [4, 5] using amplitudes computed in Refs. [6-17]. These amplitudes have been also used to obtain matched NLO plus shower predictions $[18,19]$. More recently they have been recomputed using for the first time an automated tool for the evaluation of both tree-level and loop amplitudes in Ref. [20]. A similar setup was also used to compute the first NLO results for $H+3$-jets in GGF [21].

Here we present some new results for $H+3$-jets at NLO to which we applied a set of ATLASlike cuts, and compare it with predictions for $H+2$-jets at NLO.

\section{Virtual amplitudes}

The virtual 1-loop amplitudes are generated and computed using the GoS AM framework [22], which combines the algebraic generation of $d$-dimensional integrands made of Feynman diagrams [23-26], with their numerical evaluation based upon integrand-reduction [27-33], as implemented in SAMURAi [32,34-36] and NinJA [32,37-39] and tensor integral calculus, as implemented in GOLEM95 [40,41].

In order to deal with the complexity of the amplitudes of the processes computed here, several improvements were introduced into GoSAM. These have been presented in the talk by Gudrun Heinrich at this conference [42]. A crucial step has been the possibility to exploit the optimized manipulation of polynomial expressions available in FORM 4.0 [43]. This brought, on the average, to an order of magnitude faster and smaller codes. In particular this new feature allows to generate codes for complicated processes like the one for $H+3$-jets that were not possible to generate with the previous version of GoSAM.

The one-loop amplitudes for Higgs production in GGF containing the effective $g g H$ coupling unavoidably lead to integrands that exhibit numerators with rank larger than the number of the denominators. We call these integrands "higher-rank". In the most general case they need special care if one wants to construct the amplitudes through a numerical code.

Indeed, in the GOSAM framework all the reduction strategies implemented in the NINJA, GOLEM95 and SAMURAI codes have been updated to treat the case of higher rank numerators. For the special case of diagrams generated applying the gluon-Higgs effective Feynman rules, it 
turns out that for any number of gluons, the potentially dangerous higher rank monomial always factorizes on a denominator of the relative diagram. By construction, this situation is suitable for the reduction with SAMURAI, see appendix in Ref. [20].

Recent further elaboration on the integrand level reduction technique, lead to the construction of the new library NINJA that, not only allows to treat in full generality integrands with rank in the numerator one unit larger then the number of denominators, but also gives faster and more stable results with respect to the reduction technique implemented in SAMURAI.

Numeral scalar loop integrals have been computed using ONELOOP [44].

\section{Setup}

We simulated proton-proton collisions at $8 \mathrm{TeV}$ center-of-mass energy. In our computation we have used the five-flavour scheme, massless $b$ quarks and a vanishing Yukawa $b b H$ coupling. We only consider the effective coupling of gluons to the Higgs boson evaluated in the infinite top-mass limit. The Higgs boson is stable with a mass of $m_{H}=126 \mathrm{GeV}$. To guarantee consistent setups, we use the cteq611 $\left(\alpha_{\mathrm{s}}\left(M_{Z}\right)=0.1298\right)$ and CT10nlo $\left(\alpha_{\mathrm{s}}\left(M_{Z}\right)=0.118\right)$ parton density functions (PDFs) to produce our main results at leading and next-to leading orders in QCD, respectively.

Scale uncertainties were determined following the common procedure applied in fixed-order calculations. Accordingly, we vary the renormalization and factorization scales, $\mu_{\mathrm{R}}$ and $\mu_{\mathrm{F}}$, by factors of 2.0 and 0.5 .

Everywhere, but in the effective coupling of the Higgs to the gluons, the renormalization and factorization scales are set to

$$
\mu_{F}=\mu_{R}=\frac{\hat{H}_{T}}{2}=\frac{1}{2}\left(\sqrt{m_{H}^{2}+p_{T, H}^{2}}+\sum_{i}\left|p_{T, i}\right|\right),
$$

where the sum runs over the final state jets. The strong coupling is therefore evaluated at different scales according to $\alpha_{s}^{2} \rightarrow \alpha_{s}^{2}\left(m_{H}\right) \alpha_{s}^{2}\left(\hat{H}_{T} / 2\right)$ for $H+2$-jets, and $\alpha_{s}^{5} \rightarrow \alpha_{s}^{2}\left(m_{H}\right) \alpha_{s}^{3}\left(\hat{H}_{T} / 2\right)$ for $H+3$ jets.

We apply a leading jet selection where the two highest $p_{T}$ jets are specified to be the tagged ones. The third jet therefore is defined as the hardest untagged jet of each event. Jets are constructed utilizing the anti- $k_{T}$ jet finding algorithm implemented in FASTJET [45-47] and a separation of $R=0.4, p_{t}^{\text {(jet) }}>30 \mathrm{GeV}$ and $\left|\eta^{\text {(jet) }}\right|<4.4$. Of course, our inclusive $H+$ jets samples fulfill the tagging jet requirements by definition; in the 3 -jets case, we provide predictions where even the first untagged jet is described at NLO accuracy.

All parton-level predictions, which we present in these proceedings, have been produced by combined generator packages. The $H+2$-jets samples were obtained with the GoSAM+SHERPA package while those describing $H+3$-jets production required the combination of contributions from GoSAM+SHERPA and the MADGraPh/DipolE/Event framework. More specifically, the $H+3$-jets samples were built from the Born plus virtual (BV) terms as provided by GOSAM+SHERPA and the dipole-subtracted real emission (RS) contribution as well as the integrated subtraction terms (I) as produced by MADGRAPH/DiPOLE/EvENT. We checked the consistency of our hybrid MC integration for $H+3$-jets on $H+2$-jets, verifying that the full cross section at NLO agrees with the corresponding result for the integration of all contributions (BVIRS) obtained GOSAM+SHERPA 


\begin{tabular}{|c|c|c|c|c|}
\hline Sample & \multicolumn{3}{|c|}{ Cross sections for Higgs boson plus } & \multirow{3}{*}{$r_{3 / 2}$} \\
\hline$K$-factor & $\geq 2$ jets & $f_{3}$ & $\geq 3$ jets & \\
\hline & & & & \\
\hline$H+2$-jets (LO PDFs) & $1.23_{-24 \%}^{+37 \%}$ & & & \\
\hline$H+3$-jets (LO PDFs) & $(0.381)$ & 1.0 & $0.381_{-32 \%}^{+53 \%}$ & $0.310_{0.278}^{0.347}$ \\
\hline$H+2$-jets (NLO PDFs) & $0.970_{-23 \%}^{+33 \%}$ & & & \\
\hline$H+3$-jets (NLO PDFs) & $(0.286)$ & 1.0 & $0.286_{-31 \%}^{+50 \%}$ & $0.295_{0.265}^{0.332}$ \\
\hline & $\mathrm{NLO}$ & & & \\
\hline$H+2$-jets & $1.590_{-7 \%}^{-4 \%}$ & 0.182 & $0.289_{-31 \%}^{+49 \%}$ & \\
\hline$H+3$-jets & $(0.485)$ & 1.0 & $0.485_{-13 \%}^{-3 \%}$ & $0.305_{0.284}^{0.307}$ \\
\hline$K_{2}, K_{3}(\mathrm{LO}$ PDFs for LO) & $\begin{array}{ll}1.29 & 0.911 \\
1.59\end{array}$ & & $\begin{array}{ll}1.27 & 0.806 \\
1.63\end{array}$ & \\
\hline$K_{2}, K_{3}$ (NLO PDFs for LO) & $\begin{array}{ll}1.64 & 1.19 \\
1.98\end{array}$ & & $\begin{array}{ll}1.70 & 1.10 \\
2.13\end{array}$ & \\
\hline
\end{tabular}

Table 1: Cross sections in pb for the various parton-level Higgs boson plus jet samples.

alone. Moreover, for $H+3$-jets we found excellent agreement between MADGRAPH and SHERPA for the $\mathrm{LO}$ cross section.

\section{Inclusive cross sections and multiplicity ratios}

Table 1 lists the inclusive jet cross sections $\sigma_{n}$ of all our parton-level calculations using the parameters and constraints as given in Sec. 3. Three jet multiplicity bins, $n=2,3,4$, are relevant to this study and given by requiring at least $n$ jets. We employ two of these to compare the results of the $H$ plus two-jet and three-jet calculations with each other. Except for the down-scale variation ( $\mu=\mu_{\mathrm{R}, \mathrm{F}} / 2$ ), we find the NLO rate effect to be substantial as indicated by the large $K$-factors. Interestingly, in all cases, they are rather similar in size for both jet bins $n=2$ and $n=3$. While the LO cross sections show a strong dependence on joint renormalization and factorization scale variations, the NLO cross section uncertainties due to these $\mu_{\mathrm{R}, \mathrm{F}}$ variations are considerably reduced to approximately $15 \%$.

Another interesting indicator that we consider at LO and NLO is the three-to-two jet cross section ratio, generally defined as $r_{(n+1) / n}=\sigma_{n+1} / \sigma_{n}$. Owing to the similar $K$-factors, it remains fairly stable at $\sim 30 \%$, roughly a factor 3 times higher than a sole $\alpha_{\mathrm{s}}$ effect. Just for completeness, we have also listed the inclusive three-jet fraction, $f_{3}$, belonging to each sample.

\section{Differential observables}

In Fig. 1 we show the LO and NLO predictions for the Higgs boson transverse momentum, $p_{T, H}$, as well as its rapidity, $y_{H}$. 

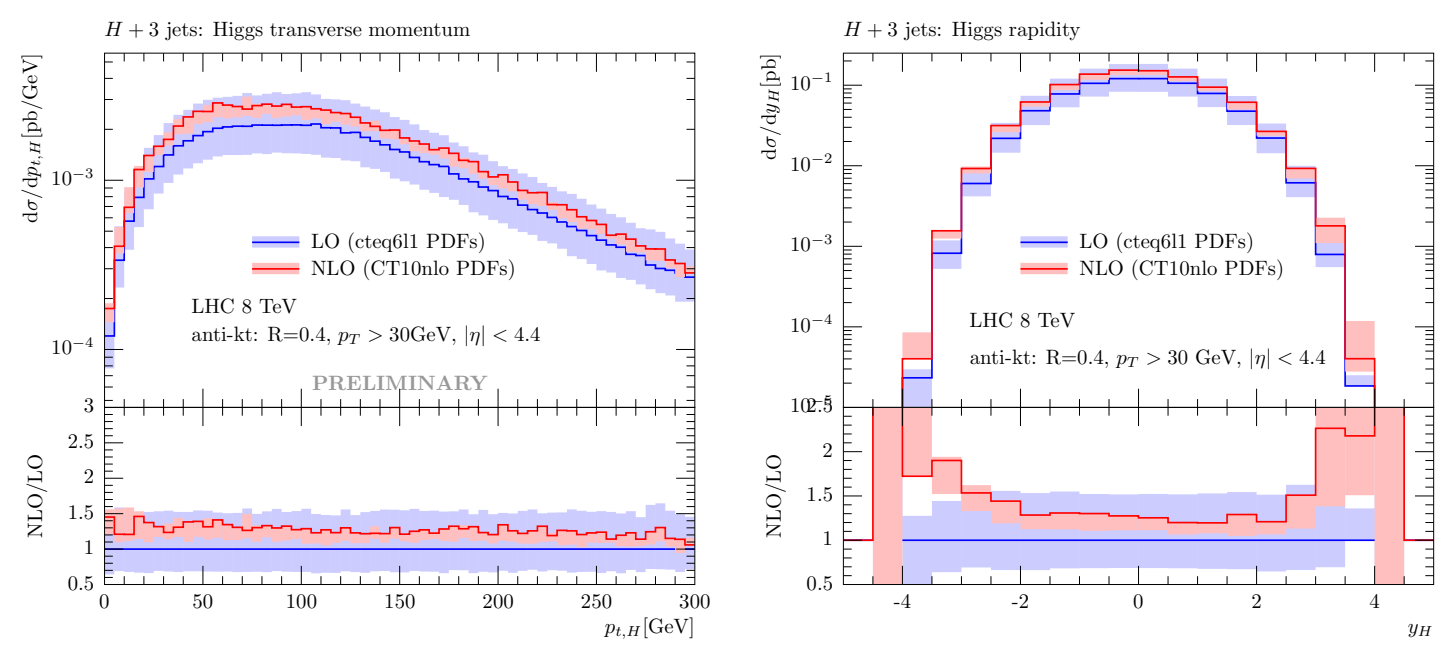

Figure 1: Higgs boson transverse momentum and rapidity distributions in $H+3$-jets production at the $E_{\mathrm{cm}}=8 \mathrm{TeV}$ LHC.

For the former observable we observe large, $\mathscr{O}(30 \%)$, positive corrections which decrease very slowly over the $p_{t}$ range plotted here. For the rapidity the corrections are spread almost uniformly over the central range and increase in the forward/backward region.

The scale uncertainties are shown by the respective envelopes of same colour. Enhancing the description to NLO accuracy, we find a reduction of these errors from $\pm 50 \%$ to less than modulus $30 \%$, which also means that the scale variation bands turn from being fairly symmetric to rather one-sided. This is a consequence of fixing the central/default scales right where the NLO cross section plateaus.
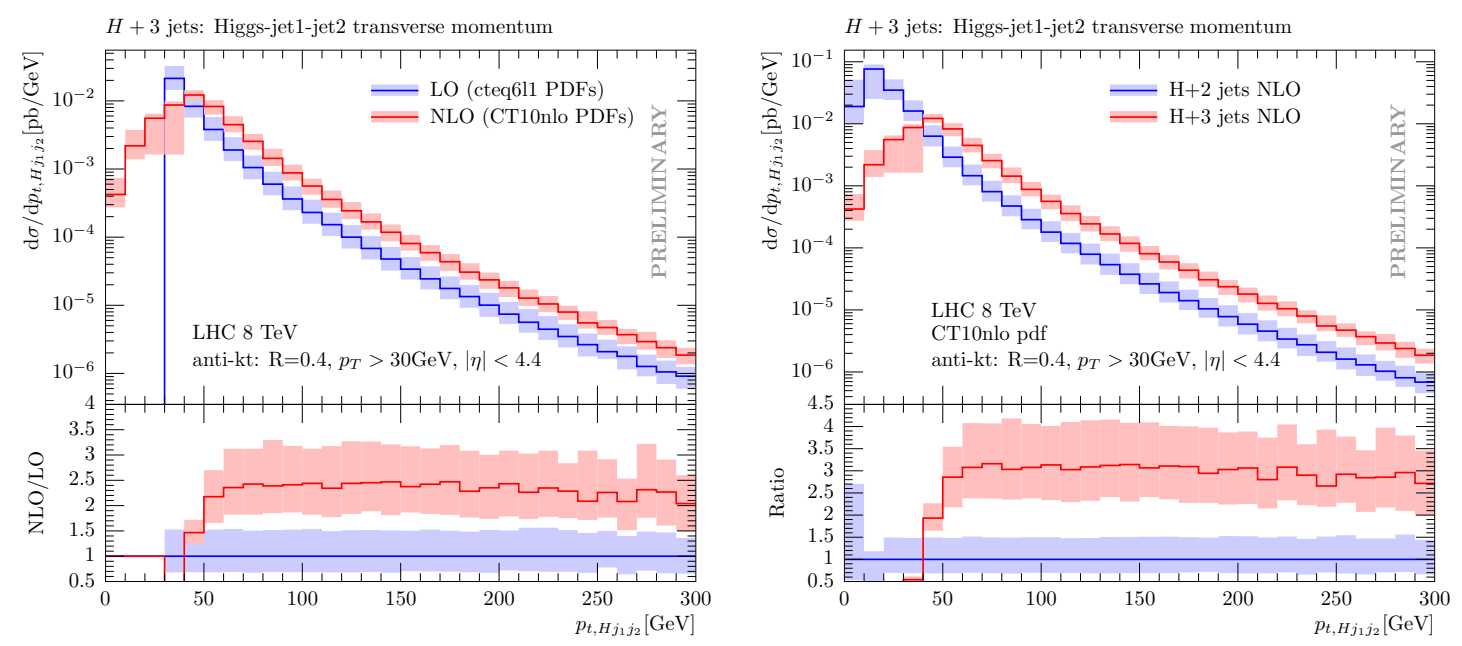

Figure 2: Transverse momentum distributions of Higgs boson plus two tagged jets in proton proton collisions at $E_{\mathrm{cm}}=8 \mathrm{TeV}$. Left: leading (blue) and next-to leading (blue) order obtained with the $H+3$-jets computation. Right: next-to leading order obtained with the $H+2$-jets computation (blu) and the $H+3$-jets computation (red).

As an example of a multi-object observable, we now discuss the $p_{T}$ distribution of the $H j_{1} j_{2}$ 
system. In Figs. 2 we show the $p_{t}$ spectrum computed from the $H+3$-jets NLO samples. First we compare it to corresponding LO predictions (Fig. 2 left), then to the ones of the $H+2$-jets NLO samples (Fig. 2 right). The $p_{t, H j_{1} j_{2}}$ is an interesting variable for our study since it is sensitive to the description of any additional parton radiation beyond the two tagging jets. It is out of question that a fixed-order description of the low $p_{t}$ region cannot be achieved reliably without supplementing the necessary resummation contributions. Nevertheless we can use this variable to point out important features of the three different calculations as well as discuss the formal accuracy of the tails of these $p_{t, H j_{1} j_{2}}$ spectra. The prediction taken from the $H+3$-jets LO sample is just given by the third-jet $p_{t}$ distribution of lowest order, with a clear indication of the jet $p_{t}$ threshold at $30 \mathrm{GeV}$.

The hardness of the third jet simply determines the recoil of the $H j_{1} j_{2}$ system. Above threshold, we therefore have a LO accurate description of the $p_{t, H j_{1} j_{2}}$ spectrum. The situation cannot be improved by an $H+2$-jets NLO computation (Fig. 2 right): the difference in the tails is caused by the replacement of LO with NLO PDFs, again pointing to a $20 \%$ effect as discussed earlier. The region below the jet threshold gets filled by real emission contributions that are too soft to be resolved as a jet. However, the resulting low- $p_{t}$ spectrum is highly unphysical due to the missing contributions from multiparton emissions. ${ }^{1}$ Turning to the NLO accurate evaluation of $H+3$ jets, we finally improve the precision to which we describe the $p_{t, H j_{1} j_{2}}$ tail. For transverse momenta larger than $60 \mathrm{GeV}$, we have achieved NLO precision. Besides neglecting Sudakov corrections, below this value we are missing contributions that only a full NNLO $H+2$-jet calculation can provide. However, contributions with two more jets beyond the tagged ones or three jets and unresolved extra emission already lead to $p_{t}$ balancing as well as $p_{t}$ enhancing effects such that we find large, $\mathscr{O}(3)$, fairly constant corrections for higher $p_{t, H j_{1} j_{2}}$ and a depletion towards zero $p_{t, H j_{1} j_{2}}$.

\section{Conclusions}

Taking advantage of the recent developments in the automated computation of NLO predictions we reported on NLO QCD results in an ATLAS-like analysis of inclusive Higgs boson plus 2-jets and 3-jets final states.

The loop amplitudes were generated with GoSAM. For tree-level amplitudes and phase space integration we used SHERPA and the MADGRAPH/DIPOLE/EvENT suite of programs. In principle, if it will be needed phenomenologically, the NLO computation including a further hard jet could be done within the same framework.

We find that NLO corrections are important and result in a substantial change of rate and jet hardness. At the level of the total inclusive cross section we find an increase of almost $30 \%$ for both $H+2$-jets and $H+3$-jets, whereas the scale variation reduces to approximately $15 \%$. We computed the $p_{t}$ distribution of the $H j_{1} j_{2}$ system including for the first time the NLO corrections.

It would be interesting to study the impact of the NLO corrections presented here when typical VBF cuts are applied. Modern Monte Carlo tools allow furthermore to study these correction in

\footnotetext{
${ }^{1}$ Known as the Sudakov effect, the very same discussion occurs, for example, for the $p_{t, H}$ distribution in Higgs boson production at NLO. The reduction seen here for the very first $p_{t, H} j_{1} j_{2}$ bin of the $H+2$-jets NLO computation is caused by those events satisfying $p_{t, H j_{1} j_{2}} \equiv 0$, i.e. Born, virtual and integrated subtraction contributions as well as those from counter events fall into the first bin diminishing the large effect from (unresolved) real emissions.
} 
a matched NLO plus parton shower framework merged with lower multiplicity predictions. These studies will be the subject of future publications.

\section{Acknowledgements}

We would like to thank Gavin Cullen for very fruitful collaboration in many phases of this project. The work of H.v.D., G.L., P.M., T.P., and V.Y. was supported by the Alexander von Humboldt Foundation, in the framework of the Sofja Kovaleskaja Award 2010, endowed by the German Federal Ministry of Education and Research. G.O. was supported in part by the National Science Foundation under Grant PHY-1068550. F.T. acknowledges partial support by MIUR under project 2010YJ2NYW. This research used computing resources from the Rechenzentrum Garching.

\section{References}

[1] Del Duca V, Kilgore W, Oleari C, Schmidt C and Zeppenfeld D 2001 Phys. Rev. Lett. 87122001 (Preprint hep-ph/0105129)

[2] Del Duca V, Kilgore W, Oleari C, Schmidt C and Zeppenfeld D 2001 Nucl. Phys. B616 367-399 (Preprint hep-ph/0108030)

[3] Campanario F and Kubocz M 2013 (Preprint 1306.1830 )

[4] Campbell J M, Ellis R K and Zanderighi G 2006 JHEP 0610028 (Preprint hep-ph / 0608194 )

[5] Campbell J M, Ellis R and Williams C 2010 Phys. Rev. D81 074023 (Preprint 1001.44 95)

[6] Del Duca V, Frizzo A and Maltoni F 2004 JHEP 0405064 (Preprint hep-ph / 0404013 )

[7] Dixon L J, Glover N and Khoze V V 2004 JHEP 0412015 (Preprint hep-th / 0411092 )

[8] Badger S, Glover N and Khoze V V 2005 JHEP 0503023 (Preprint hep-th/ 0412275 )

[9] Ellis R K, Giele W and Zanderighi G 2005 Phys. Rev. D72 054018 (Preprint hep-ph/ 0506196 )

[10] Ellis R K, Giele W and Zanderighi G 2006 Phys. Rev. D73 014027 (Preprint hep-ph / 0508308 )

[11] Berger C F, Del Duca V and Dixon L J 2006 Phys. Rev. D74 094021 (Preprint hep-ph / 6008180 )

[12] Badger S and Glover N 2006 Nucl. Phys. Proc. Suppl. 160 71-75 (Preprint hep-ph / 0607139)

[13] Badger S, Glover N and Risager K 2007 JHEP 0707066 (Preprint 0704 . 3914)

[14] Glover N, Mastrolia P and Williams C 2008 JHEP 0808017 (Preprint 0804 . 4149)

[15] Badger S, Glover N, Mastrolia P and Williams C 2010 JHEP 1001036 (Preprint 0909.4475 )

[16] Dixon L J and Sofianatos Y 2009 JHEP 0908058 (Preprint 0906.0008 )

[17] Badger S, Campbell J M, Ellis R K and Williams C 2009 JHEP 0912035 (Preprint 0910 . 4 481)

[18] Campbell J M, Ellis R K, Frederix R, Nason P, Oleari C et al. 2012 JHEP 1207092 (Preprint 1202.5475)

[19] Höche S, Krauss F and Schönherr M 2014 (Preprint 1401. 7971)

[20] van Deurzen H, Greiner N, Luisoni G, Mastrolia P, Mirabella E et al. 2013 Phys. Lett. B721 74-81 (Preprint 1301.0493) 
[21] Cullen G, van Deurzen H, Greiner N, Luisoni G, Mastrolia P et al. 2013 Phys. Rev. Lett. 111131801 (Preprint 1307.4737)

[22] Cullen G, Greiner N, Heinrich G, Luisoni G, Mastrolia P et al. 2012 Eur. Phys. J. C72 1889 (Preprint 1111.2034)

[23] Nogueira P 1993 J. Comput. Phys. 105 279-289

[24] Vermaseren J A M 2000 (Preprint math-ph/0010025)

[25] Reiter T 2010 Comput. Phys. Commun. 181 1301-1331 (Preprint 0907.3714 )

[26] Cullen G, Koch-Janusz M and Reiter T 2011 Comput. Phys. Commun. 182 2368-2387 (Preprint 1008.0803)

[27] Ossola G, Papadopoulos C G and Pittau R 2007 Nucl. Phys. B763 147-169 (Preprint hep-ph/0609007)

[28] Ossola G, Papadopoulos C G and Pittau R 2007 JHEP 0707085 (Preprint 0704 . 1271)

[29] Ellis R K, Giele W T and Kunszt Z 2008 JHEP 03003 (Preprint 0708 . 2398)

[30] Ossola G, Papadopoulos C G and Pittau R 2008 JHEP 0805004 (Preprint 0802 . 1876)

[31] Mastrolia P, Ossola G, Papadopoulos C and Pittau R 2008 JHEP 0806030 (Preprint 0803 . 3964)

[32] Mastrolia P, Mirabella E and Peraro T 2012 JHEP 1206095 (Preprint 1203.0291 )

[33] Mastrolia P, Mirabella E, Ossola G and Peraro T 2012 Phys. Lett. B718 173-177 (Preprint 1205.7087)

[34] Mastrolia P, Ossola G, Reiter T and Tramontano F 2010 JHEP 1008080 (Preprint 10 06.0710 )

[35] Mastrolia P, Mirabella E, Ossola G, Peraro T and van Deurzen H 2012 PoS LL2012 028 (Preprint $1209.5678)$

[36] van Deurzen H 2013 Acta Phys. Polon. B44 2223-2230

[37] Peraro T to appear.

[38] van Deurzen H, Luisoni G, Mastrolia P, Mirabella E, Ossola G et al. 2013 (Preprint 1312 . 6678)

[39] Peraro T 2013 Acta Phys. Polon. B44 2215-2221

[40] Cullen G, Guillet J, Heinrich G, Kleinschmidt T, Pilon E et al. 2011 Comput. Phys. Commun. 182 2276-2284 (Preprint 1101.5595)

[41] Guillet J P, Heinrich G and von Soden-Fraunhofen J 2014 Comput. Phys. Commun. 185 1828-1834 (Preprint 1312.3887)

[42] Heinrich G et al. Automated one-loop calculations with GoSam 2.0, these proceedings.

[43] Kuipers J, Ueda T, Vermaseren J and Vollinga J 2013 Comput. Phys. Commun. 184 1453-1467 (Preprint 1203.6543)

[44] van Hameren A 2011 Comput. Phys. Commun. 182 2427-2438 (Preprint 1007.4716)

[45] Cacciari M and Salam G P 2006 Phys. Lett. B641 57-61 (Preprint hep-ph/0512210)

[46] Cacciari M, Salam G P and Soyez G 2008 JHEP 0804063 (Preprint 0802 . 1189)

[47] Cacciari M, Salam G P and Soyez G 2012 Eur. Phys. J. C72 1896 (Preprint 1111. 6097) 